\title{
Automatic MRI Brain Tumor Segmentation Techniques: A Survey
}

\author{
Otman Basir ${ }^{1}$ \& Kalifa Shantta ${ }^{2 \#}$ \\ ON. Canada. \\ \#corresponding author \\ Type of Work: Peer Reviewed. \\ DOI: 10.21013/jas.v16.n2.p2 \\ DOI URL: https://dx.doi.org/10.21013/jas.v16.n2.p2
}

${ }^{1,2}$ Electrical and Computer Department, University of Waterloo, 200 University Ave W, Waterloo,

\begin{abstract}
How to cite this paper:
Basir, O., Shantta, K. (2021). Automatic MRI Brain Tumor Segmentation Techniques: A Survey. IRA-International Journal of Applied Sciences (ISSN 2455-4499), 16(2), 25-38. DOI:

https://dx.doi.org/10.21013/jas.v16.n2.p2
\end{abstract}

(C) IRA Academico Research.

(cc) BY-No 4.0 International License subject to a proper citation to the publication source of the work.

Disclaimer: The scholarly papers as reviewed and published by IRA Academico Research are the views and opinions of their respective authors and are not the views or opinions of IRA Academico Research. IRA Academico Research disclaims of any harm or loss caused due to the published content to any party.

IRA Academico Research is an institutional publisher member of Publishers International Linking Association Inc. (PILA-CrossRef), USA. IRA Academico Research is an institutional signatory to the Budapest Open Access Initiative. Hungary advocating the open-access of scientific and scholarly knowledge. IRA Academico Research is a registered content provider under Open Access Initiative Protocol for Metadata Harvesting (OA/-PMH).

The journal is indexed \& included in WorldCat Discovery Service (USA), CrossRef Metadata Search (USA), WorldCat (USA), OCLC (USA), Open J-Gate (India), EZB (Germany) Scilit (Switzerland), Airiti (China), Bielefeld Academic Search Engine (BASE) of Bielefeld University, Germany, PKP Index of Simon Fraser University, Canada. 


\begin{abstract}
Image segmentation plays a crucial role in recognizing image signification for checking and mining medical image records. Brain tumor segmentation is a complicated assignment in medical image analysis. It is challenging to identify precisely and extract that a portion of the image has abnormal tissues for further diagnosis and analysis. The method of segmenting a tumor from a brain MRI image is a highly concentrated medical science community field, as MRI is non-invasive. In this survey, brain MRI images' latest brain tumor segmentation techniques are addressed a thoroughgoing literature review. Besides, surveys the several approved techniques regularly applied for brain tumor MRI segmentation. Also, highlighting variances among them and reviews their abilities, pros, and weaknesses. Various approaches to image segmentation are described and explicated with the modern participation of several investigators.
\end{abstract}

Keywords: Magnetic Resonance Imaging, Atlas-based Segmentation, Classification, Thresholding Technique, Watershed Segmentation, Region growing.

\title{
INTRODUCTION
}

Medical Imaging can be defined as creating an image of a human body's internal parts, subsequently being analyzed for medical and clinical purposes. Another application of this procedure can be the acquisition of knowledge of human tissues and organs. Numerous techniques may be used to obtain brain images. These include computed tomography (CT), functional MRI (fMRI), magnetic resonance image (MRI), single-photon emission and computed tomography (SPECT), and X-radiation (X-ray). Furthermore, segmentation occupies a critical function for many applications in analysis, extraction of features, image understanding, and interpretation. It also has extensive medical science uses such as atlas matching, blood-cell delineation, tumor volume estimation, image matching registration, surgical preparation, tissue classification, and tumor localization [1].

\section{Original MR}

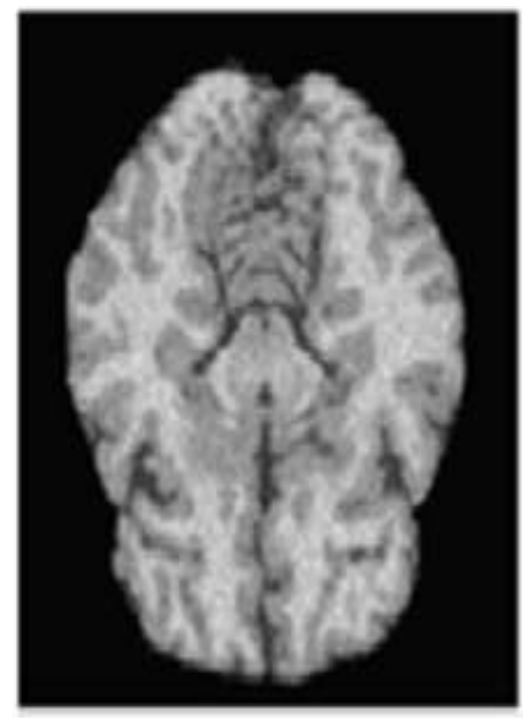

Segmented Image

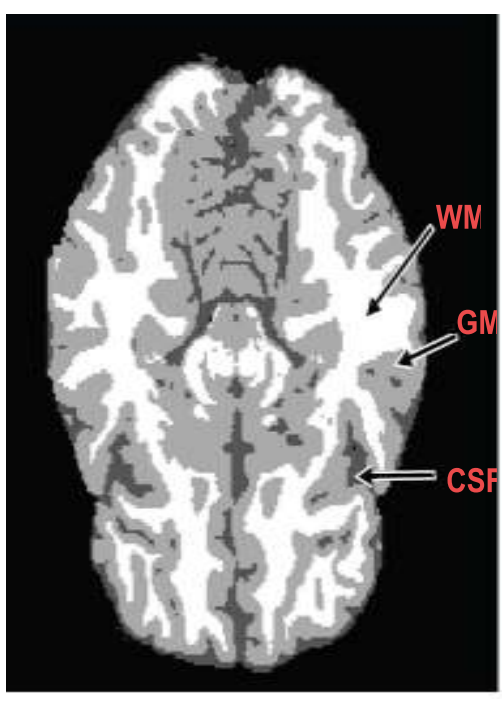

Fig. 1: Brain MRI and segmented picture with three markers: WM, GM, and CSF.

Brain MRI segmentation impacts all the analysis results; therefore, this is a necessary exercise in several clinical applications. These various processing stages are dependent on the precise segmentation of anatomical areas. A regular MRI segmentation is made for delineating lesions, the visualization, and measurement of other brain structures, surgical planning, and image-guided interventions. Numerous segmentation methods have resulted from these diverse imaging processing techniques, which vary in complexity and accuracy. There are many types of areas in the human brain, three of the principal ones being: cerebrospinal fluid (CSF), grey matter (GM), and white matter (WM), as shown in Figure 1. The majority of medical imaging segmentation procedures' principal aim is to extract these areas and emphasize them as required. Image segmentation can be described as segregating an image into 
various areas based on the digital images intensities and set of pixels. Each area distinguished from others depends on its attributes, such as colour, intensity, and texture. Image segmentation methods were applied to extract the CSF, GM, and WM [2,3], whereas different studies can segment tumor detection in MRI brain images [4,5]. A brain tumor can be defined as developing abnormal brain cells, which could be malignant or benign. A benign tumor structure is uniform and has no active cancer cells. However, a malignant tumor does contain cancer cells. Meningioma's and gliomas, categorized as benign tumours, are instances of a low-grade tumour, resemble normal brain cells, and rise gradually. However, astrocytoma and glioblastoma are in a high-grade tumor category of malignant tumor and tend to overgrow [6]. Brain MRI segmentation, a necessary initial stage, has numerous neurology applications, including functional imaging, operational planning, and quantitative analysis. Even though MRI can define the brain structures precisely, medical image segmentation is a demanding assignment. The reason for this is low contrast, ill-defined boundaries, poor spatial resolution, inhomogeneity, the variability of object shapes, noise, partial volume impact, and certain other acquisition items within the retrieved data, in addition to lack of complete anatomy paradigms.

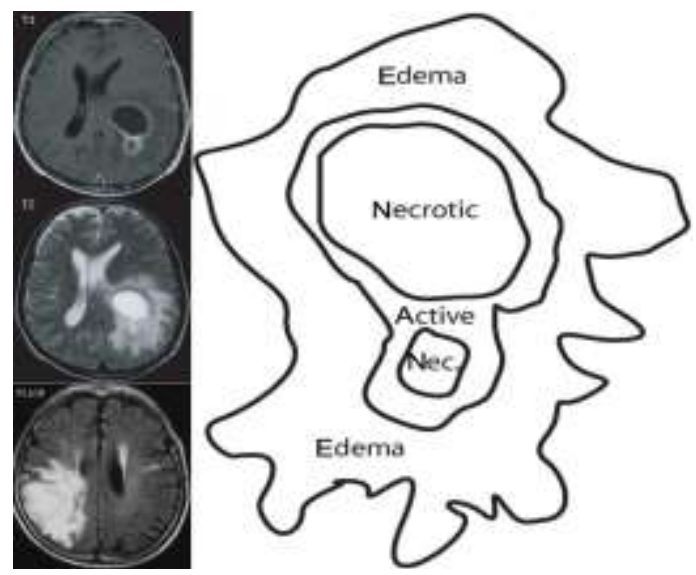

Fig. 2: Left is three types of brain tumor MRI images: T1 with contrast, T2 and FLAIR image; right is three main components after segmenting brain tumor[7].

Brain tumor segmentation is to segment uncommon tissues such as active cells, necrotic core, and Edema from joint brain tissues containing GM, WM, and CSF. [7]. Figure 2 shows three brain tumor T1 with contrast, T2, and FLAIR images with three main components after segmenting brain tumor. Since brain tumor segmentation regularly involves massive amounts of data, it is among the most demanding and crucial assignments in several medicalimage applications. Artefacts caused by acquisition insufficient time, patients' motion, and soft-tissue margins usually are poorly drawn. Furthermore, a complete classification of various kinds of the tumor is of multiple sizes or shapes that may be apparent in different image intensities. Moreover, some of these can influence the surrounding frameworks that transform the picture's depth in the tumor's vicinity. Furthermore, the World Health Organization (WHO) indicates that, from a global perspective, approximately 400,000 people have a brain tumor, 120,000 of whom died during the previous year [8]. Before brain surgery, chemotherapy or radiotherapy is considered; medical practitioners must verify the brain tumor's boundaries and areas and establish its exact location and the affected region. To assess cancer's detrimental impact, the instrument for brain tumor segmentation may be either automatic or semi-automatic, also serving as a necessary stage, enabling doctors to recognize the brain tumor before considering brain surgery. In the last years, numerous methods based on the tissue characteristic have been improved for MRI segmentation [9].

Olabarrge et al. [10] and Yo [11] categorize brain tumor segmentation techniques into three classes based on the level of essential human interaction: manual, semi-automatic, and fully automatic. In the coming sections, there is a description of each category regarding significant benefits and disadvantages.

\section{MANUALSEGMENTATION}

The manual method requires drawing the tumor's boundaries and frameworks of interest manually or depicting the area of anatomic frameworks labelled differently [11]. In this technique, persons with expertise, such as anatomists, qualified technologists, and radiologists, apply the image's information and utilize further knowledge. In addition, to 
ease drawing areas of interest and image portrayal, manual depiction needs software tools with complex graphical user interfaces, from a practical aspect. The tumor selection in the region of interest (ROI), monotonous, and assignments occupy a considerable amount of time. Figure 2 highly specialized persons conducted a hand segmentation of glioma on the same picture and person [12]. There were significant variations in each expert's resulting segmentation. Since MRI equipment produces various two-dimensional cross-sections, the knowledgeable person must examine the dataset slice-by-slice to select those best illustrative of the appropriate areas and delineate them meticulously [13].

Furthermore, brain tumor manual segmentation is usually undertaken according to a single image having a greater intensity supplied by an injected contrast agent [14]. Nevertheless, suppose someone who is not an anatomist, trained technologist, or radiologist but familiar with brain anatomy draws the area of interest. In that case, poor segmentation results will probably be produced.

\section{SEMI-AUTOMATIC SEGMENTATION}

Human operators are frequently required to intervene to commence the technique of semi-automatic brain tumor segmentation and verify the authenticity of the result or even correct it manually. Current research generally supports semi-automatic segmentation of brain tumor to reduce human intervention to a minimum. However, Olabarriaga et al. [15] state that the interacting brain cancer segmentation techniques' principal parts are the computation and communication components and the operator interface. The computational component matches at least one part of a program that can depict the tumor, given some criteria. The interacting component mediates information between the computational component and the user.

Furthermore, it converts the computational component's results into observed feedback for the operator and that user's data input into criteria for the program. Moreover, the user and the computer's announcement are performed through the input, output tools, and regulated by the operator screen. The visual information portrayed on the display monitor is analysed by the user, who reacts appropriately, giving the computation feedback.

\section{FULLY AUTOMATIC SEGMENTATION}

When fully automatic techniques are applied, the segmentation of the tumor is established with no personal interaction. Previous knowledge of algorithms and human intelligence is usually combined in these techniques and is typically developed by applying model-based and soft-computing methods, such as deformable paradigms. The next section will review the soft-computing strategies. The process of studying automatic brain tumor segmentation, which indicates a difficulty that humans can resolve successfully, corresponds to intriguing research in pattern detection and machine learning. Nevertheless, it remains an exact challenge to develop precise automatic techniques. This is clarified because humans need to apply top pictorial processing and specific field information to undertake this assignment [15], thereby developing fully automatic strategies to be exceptionally problematic.

\section{SEGMENTATION TECHNIQUES}

Several researchers have categorized image segmentation schemes under various headings, which are considered in $[16,17]$. These schemes are region and threshold-based, as well as pixel categorization and model-based methods. Other classifications presented in the references $[18,19]$ are threshold, region, and boundary-based techniques. Although several brain tumor division techniques exist, No primary segmentation method exists to provide a satisfactory outcome for every imaging application. However, techniques are frequently optimized to manage particular imaging modalities, for example, magnetic resonance imaging. Various authors have generally classified segmentation methods into three significant categories: Threshold-based approaches, region-based approaches, and pixel classification approaches [20, 21, 22]. Figure 3 reviews the various MRI segmentation approaches [23]. 


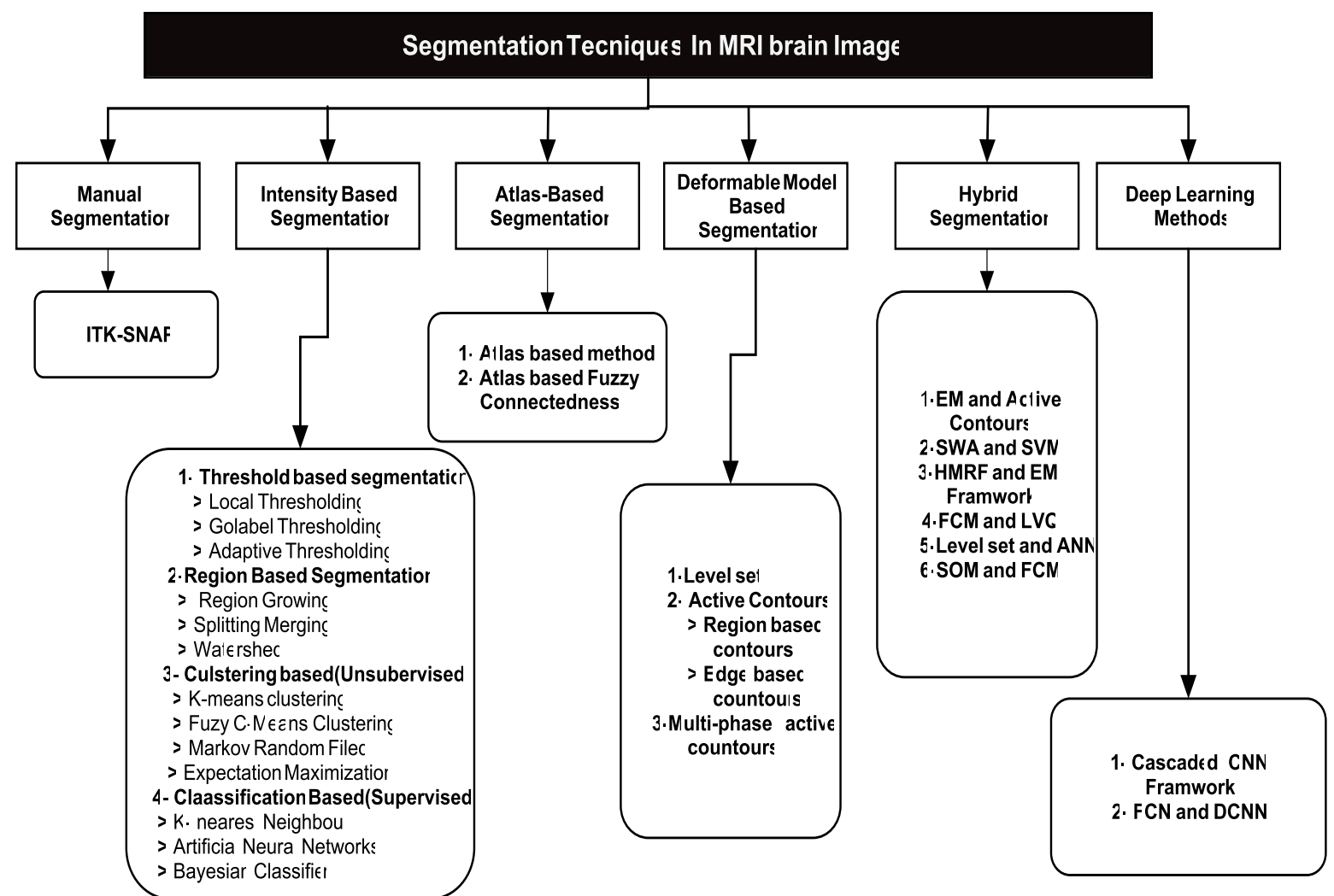

Fig. 3: Several segmentation methods in MRI brain image examination[23].

\section{THRESHOLDING}

Thresholding is rapid, simple, and easy to apply, is among the most basic image segmentation methods. It functions based on transforming a scalar image into a binary one. Edge evaluation is computed according to the image strength values and the pixels' strength values compared with the threshold value. Value 1 is allocated to pixels having an intensity value equal to or above the threshold value. However, lower intensity pixels are marked zero, thereby segregating the background (dark pixels) and the foreground (white pixels) and region.
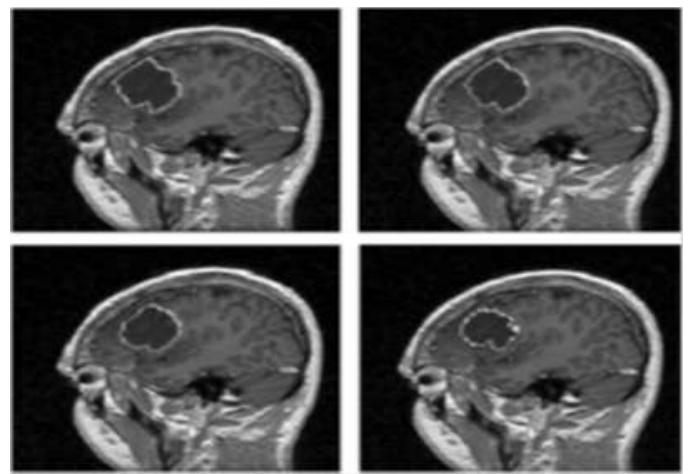

Fig. 4: Hand segmentation by diverse specialists of glioma on the MRI [12].

Regarding the grayscale picture of the original; for example, $f(i, j)$, the primary threshold value $\mathrm{T}$ is selected according to the intensity values. The image is then divided into two $\mathrm{H} 1$ and $\mathrm{H} 2$ sets in which $\mathrm{H} 1$ and $\mathrm{H} 2$ contain pixel sets lighter and darker than the value of the threshold. Furthermore, the mean intensities h1 and h2 of H1 and $\mathrm{H} 2$ are computed according to an estimate of a new threshold value. 
$T_{1}=\frac{h_{1+h_{2}}}{2}$ if $\left\|T-T_{1}\right\| \geq \Delta T(1)$

$h(i, j)= \begin{cases}1 & \text { if } f(i, j) \geq T_{F} \\ 0 & \text { if } f(i, j)<T_{F}\end{cases}$

The final value when one global threshold is selected for the whole picture, this is known as a global threshold. This remarkably intuitive image thresholding method is straightforward to calculate and not combine any local pixel association. It is perfect for segmenting images without any fixed shapes because no previous knowledge is required.

The Otsu technique attempts to obtain the maximum value for the global threshold to isolate the object from the image context [24]. In this method, it is presupposed that the histogram is bimodal. However, this technique will not succeed if the two classifications' sizes differ or if the illumination in the image's vicinity varies. Sujan et al. [25], applied Otsu's thresholding together through the logic operator, for example, erosion and dilation, to recognize brain tumor from MRI images. In [26], attempted to locate a global threshold by applying both tumor and non-tumor region segmentation level sets. Only the zero levels are required to be fed into this to perform the process. However, if the intensity levels in the tumor and non-tumor regions differ, its usefulness becomes uncertain. Furthermore, the global thresholding technique's effectiveness declines when the picture pixels' strength is low-contrast, nonhomogeneous, or at a high noise level; neither can the picture be divided into two regions areas by applying one edge value. An image can contain two or more places where the substances do not portion equal strength values. In a situation of this kind, several threshold values are applied to separate a picture into different areas of interest.

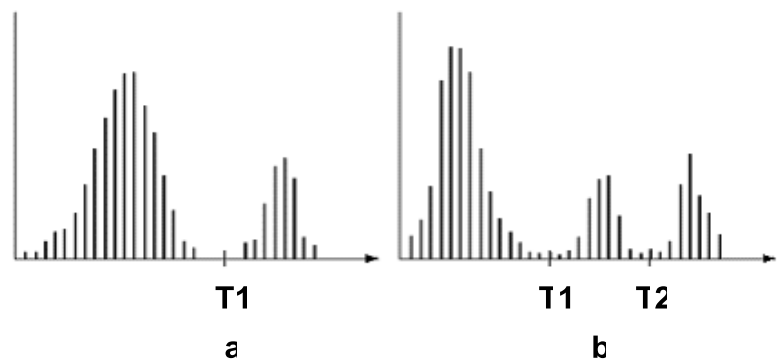

Fig.5: Gray-level histogram that can be partitioned by (a) single threshold,(b)multiple thresholds.

Figure 5 shows Gray-level histogram that can be partitioned by single threshold and multiple thresholds. The literature advocates numerous thresholding methods for complex and local thresholds, which satisfy the standard [27]. Such techniques are perfect for segmentation when it is impossible to forecast one threshold from the image histogram. Thresholding is regularly applied as a pre-processing stage to complicated segment pictures; for example, MRI, because they cannot utilize all the appropriate data from the image.

\section{REGION GROWING}

The most basic regionally grounded segmentation method is the area growing, as applied to remove an associated area of like pixels from a picture. Region growing begins with a minimum of a single seed, which is part of the region of interest structure. Furthermore, adjacent seeds are authenticated, and the ones that meet the similarity requirements are added to the area. The resemblance standard is decided by a scope of pixel strength amount or extra factors within the picture. The seeds may be either supplied by an automatic seed-finding process or selected manually. The cycle repeats itself up to the point where no other pixels may be included in the area. The process of region growing is an essential yet vital image segmentation technique used for homogeneous segment areas having equal intensity values. It needs no implementation or knowledge of the previous shape of any object with a different form. The principal working criteria require that each pixel should be located in an area and that they should be connected, and comply with certain specific similarity conditions, with components being disjointed. In addition, the two different regions should not have identical characteristics [28]. It commences with a seed value located in one area of interest, which may be chosen automatically or manually. Following this, the adjacent neighbourhoods or pixels are connected to the seed point in conformity with certain predefined similarity principles. 

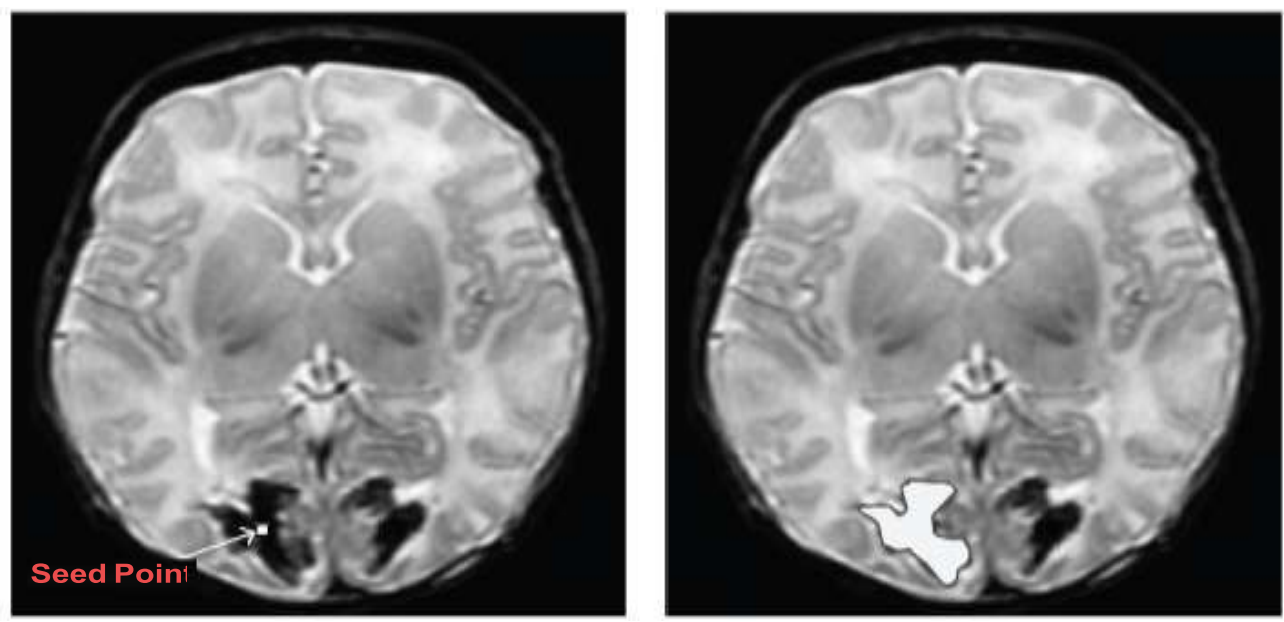

Fig. 6:A seed point is manually selected in ROI. Final segmentation region.

Figure 6 shows the region growing applied to MRI image. This technique continues to proceed until every pixel is categorized into one area, thereby sustaining all pixels' connectivity developed from the seed point, which assimilates local connections among pixels. Although its integral easiness improves this technique, it remains sensitive to noise and seed initialization. However, concerning images where adjacent areas share like intensities and non-smoothly differing areas such as textured pictures, it performs poorly. This may be used for pictures impacted by different intensities of lighting, but after appropriate pre-processing.

Furthermore, partial volume impact is a significant element that restricts segmentation precision because it obscures the intensity differentiation at the boundary of both types of tissue; consequently, causing a voxel to represent at least two tissue types [29]. To surmount this difficulty, Modified Region Growing SegmentationMethods (MRGM) is implemented by applying gradient information to recognize the borders [30]. A relative analysis of MRGM and the conventional area is growing, as indicated in [31], thereby verifying MRGM to give enhanced cancer segmentation results in three-dimension T1 MRI images.

\section{WATERSHED}

The behaviour of water in a landscape can be a useful metaphor for explaining the fundamental watershed segmentation technique. During rainfall, water drops will descend into various areas, following the landscape downhill, and resulting in the water being at the lowest point in the valleys. Consequently, every valley will have an area into which all the water drains, meaning that every valley is linked with a catchments basin, with every location within the landscape belonging to a single basin. Moreover, at locations at which water emerging from various basins coincides, dams will be constructed, and once the water scale reaches its most in the landscape, the procedure is terminated. Consequently, the landscape is separated into areas divided by dikes, known as watersheds or watershed lines. This produces completes the pictures, thereby averting the necessity for any type of contour connection. In the brain, tumor segmentation watershed is widely applied in this field. In [32,33], Brain tumor segmentation is an offer by using several -scale watershed conversion. The watershed algorithm might be used on the gradient image instead of the original image. Figure 7 shows the watershed applied to the blobs image. In [33], performed communicating approach for Magnetic Resonance Imaging brain lesion division forms mass at various measures where the operator can choose and unchosen to model the wanted anatomic subject. In [34], in this study, tumor from MRI images was segmented using the watershed algorithm. The outcomes assessment pointed out that there is an improvement in the segmentation period and the accuracy compared to hand-operated segmentation. The examination similarly recognized some crashes in the watershed method, as boundaries were badly represented in the data, and showed the direction in the hand-operated segmentation outcomes proceeding regularly bigger segmentations. The examination asked issues about the sense of employing skilled segmentations to determine the ground truth. In [35], performed a sigs- based on enhanced watershed method employing the previous data of the 
examination photos into the partition of a brain tumor; the seeded zone -increasing approach was applied for the tag of the region of interest.

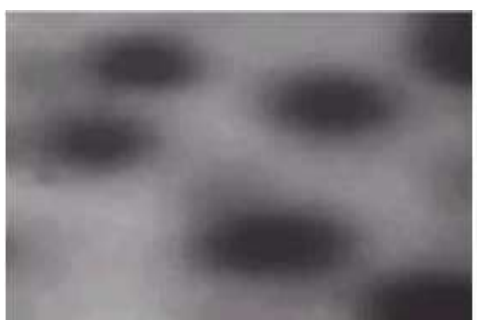

Image of blobs

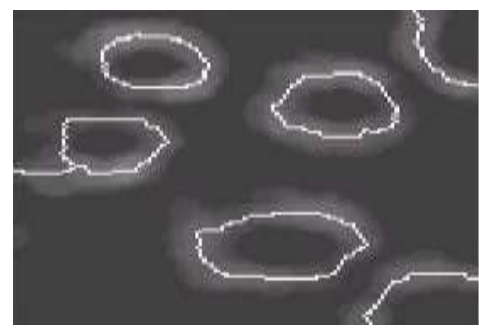

Watershed lines

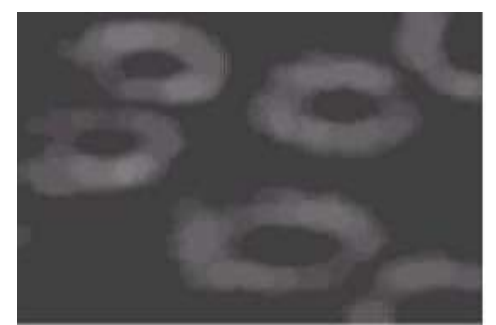

image gardient

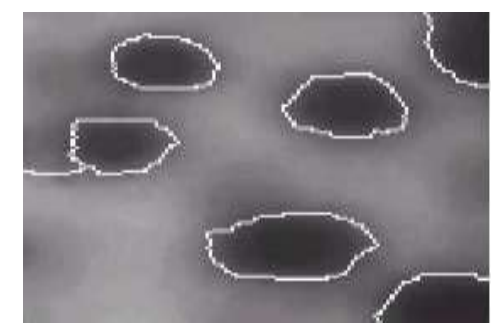

Watershec lines superimpos $\epsilon \mathrm{d}$ on

original image

Fig. 7: Watershed segmentation on image of blobs

In [36], a watershed segmentation method applied to distinguish tumor in two and three-dimension brain Magnetic resonance imaging. A simplistic managed block and the image-based method were applied to examine MRI brain photos with comparatively inexpensive computation time demands.

\section{PIXEL CLASSIFICATION METHODS}

A further kind of segmentation technique is based on pixel categorisation. Pixel characteristics may be applied in order to represent pixels in an image, which could comprise local texture, grey level and colour components for every pixel. Pixel categorisation in a one-channel (or one-frame) picture is generally based on grey level; moreover, it is possible to undertake image segmentation in a single-dimensional factor space. However, in the case of multispectral (multimodality) or multichannel (multiple-frame) images, segmentation may be undertaken in multidimensional feature space. Furthermore, the techniques used in brain tumor division, which are grounded on pixel categorisation, are restricted to the application of monitored or unmonitored algorithms to group pixels in the factor space. Clustering involves grouping related things into one group, whereas things having unrelated characteristics are grouped into various groups based on some likeness measures. The likeness is calculated as a suitable distance measure, whereas it is clear that a correspondence amount is the distance that separates two vectors within the attributes map as shown below:

$d=\left(\sum_{k-1}^{n}\left\|x_{i}-x_{j}\right\|^{p}\right)^{1 / p}$

Where $x_{i}-\left(x_{i}{ }^{l}, \ldots, x_{i}^{n}\right) \mathrm{R}^{\mathrm{n}}$ and $x_{j}-\left(x_{j}{ }^{l}, \ldots, x_{j}^{n}\right)$.The two vectors in the feature space are denoted $\mathrm{R}^{\mathrm{n}}$. If $p=1$ it related to Mahalanobis distance, and $p=2$ related to Euclidean distance.

$n d\left(x_{i}, x_{j}\right)=\frac{x_{i}^{T} x_{j}}{\left\|x_{i}\right\|\left\|x_{j}\right\|}$

The transpose operation is $\mathrm{T}$. This quantity gives data on the cosine among the vectors $x_{i}$ and $x_{j}$ within the attribute domain. Every group is indicated by its average and contrast, which shows the formation of clusters and the compactness of the entities in the group; moreover, it is optimized in accordance with a cost function, which usually considers the resemblance within each of the clusters and the difference between them. The literature suggests numerous clustering methods, of which the fundamental ones include unsupervised techniques, for example: FCM, k-means and arithmetical techniques as MRF. The monitored techniques contain Bayes and artificial neural networks [37]. 


\section{FUZZY C-MEANS}

In numerous circumstances, it is difficult to decide whether a pixel ought to be part of an area because the features that establish homogeneity could have no have sharp transitions at area borders [37]. However, fuzzy set ideas may be submitted into the segmentation procedure as in the C-mean method to relieve this predicament. The region of unsupervised image segmentation by pixel categorization. The FCM clustering is particularly favoured, especially in brain tumor segmentation [38,39]. This initial stage in applying the FCM technique to brain tumor segmentation involves the establishment of tissue classifications. Subsequently, every pixel is allocated membership values to the tissue classifications depending on its characteristics, such as texture and intensity. The fuzzy membership functions, that are restricted to being between zero and one, indicate the resemblance levels among the information amount at a particular place as well as the typical data value, or centroid, of its classification. Consequently, a fuzzy set amount close to the number one indicates that the information value at the said place is near the centroid of the group. Moreover, the procedure converges more quickly and the grouping outcomes are enhanced if the initialisation is performed by estimating the cluster centre precisely. In [39], used the splitting method of discrete curve evolution (DCE) for locating the optimal assessment of cluster centres for different MR head appearance division. Since the suggested method shortened the convergence time, inexpensive computational time resulted. It acts on the policy of selecting membership values $u_{a b}$ to every attribute data point $x_{a}, A=1,2, \ldots, n, x_{a} \in \mathbb{R}^{\mathrm{d}}$ identical to the cores of the groups $c_{b}, j=1, \ldots, \ldots, z$, and $z<m$ based on the Euclida measure. Belonging values meet the next characteristics:

$$
\begin{aligned}
& \sum_{b=1}^{z} u_{a b}=1, \text { for all } a,(5) \\
& \sum_{b=1}^{m} u_{a b}<n, \text { for all } b .(6)
\end{aligned}
$$

$$
0 \leq u_{a b}<1 \text { for all } a b
$$

The broader membership amount means a higher similarity to refer to the appropriate group. One bit of data may relate to a couple or extra collections. The fundamental group amounts are appointed to precise data point $x_{a}$ to refer to a group b. Next, the cores of the groups and the belonging values are refreshed. The fuzzy separating is completed over repeated minimization of the actual role. The procedure stops after the belonging values in sequential repetition are nearly enough. In [37], outlined the regular FCM algorithm combining spatial contextual data, next named FCM_S, applying the adjusted actual role to regain strength inhomogeneity and recognizing the tags to be affected by those of their direct adjacent illumination. Nevertheless, it calculates the neighbourhood term at every repetition, which affects significantly in execution time. To defeat this difficulty, the mean and median filtering is applied to change FCM_S to FCM_S1 and FCM_S2 [40]. Benaichouche et al. [41], also decreased the computational time by applying EnFCM by functioning on a gray-scale histogram quite than photovoxels. Forouzanfar et al. [42], showed a neighbourhood attraction conditioned on neighbouring pixels' related position and features. FLICM procedure that includes the local locative data and gray scale data in a fuzzy method was explained in [43]. Its achievement is separate from the form of noise. The adaptive monitoring parts incorporated in the objective function are obtained in an automatic way in the absence of any factor assortment such as $\alpha$, giving the segmentation mode crispiness. Song [44] introduced enhanced segmentation precision by mixing modified spatial limitations within the middle pixel and adjacency pixels in correlation determination. Ramakrishnan [45], introduced the segmentation of Magnetic resonance imaging images utilizing Artificial Bee Colony with suitability task provided simultaneously through fuzzy c-means grouping in [46]. It is not simply divisions the cancer then additionally presents its strength. Neighborhood intuitionistic fuzzy cc-means clustering algorithm with a genetic algorithm(NIFCMGA) is suggested in [47], which supports the assessment of the optimal parameters for IFCM and reduces noise and outliers over adjacency belonging. Excellence in division outcomes of MRI images in noise and idiopathic intracranial hypertension $(\mathrm{IIH})$ has been reached in [48] by combining a limited variable with every intensity, which represents its importance in making the local relationship values of the groups. Nevertheless, rising idiopathic intracranial hypertension scales cannot be deal with this method. Integration of factor context data and Gaussian role into the objective process in [49] has demonstrated profits in IIH estimation and bias field adjustment.

\section{MARKOV RANDOM FIELDS}

The majority of clustering techniques regard neither locative data nor the dependence among the pixels on the picture's superficial. The unmonitored grouping technique proposed by Markov random fields (MRF) supplies a method of assimilated locative data into the clustering procedure. In some circumstances, this decreases the potential difficulty of overlapping clusters and the impact of noise on the gathering outcome [50]. However, in brain tumor segmentation, if strength is definitively designated as a lesion (non-tumor), this implies that adjacent areas will also show a lesion (non-tumor) tag. Consequently, this has encouraged several academics to utilize conditional random 
fields (CRFs) and even Markov random domains for different segmentation assignments. Furthermore, CRF and MRF can represent complicated dependencies between data instances, thereby resulting in high precision in brain tumor segmentation assignment. In [51], offered an unsupervised method employing an MRF paradigm arithmetical applied impacts that adjacent pixels should have on every other's tags eliminating the necessity of logic operator. They presumed that the tissue classes in different Magnetic Resonance Imaging: cerebrospinal fluid, GM, WM, cancer, and brain swelling may be demonstrated through a combination paradigm of Gaussians, and learned the MRF through the repeated condition methods procedure. In [52], suggesting a process that distinguishes irregularities in the brain utilizing a several layer MRF agenda. The data layers involved voxel strengths, thematic consistency, locative positions, and operator input. This study recognized that a stated pixel would alter its highgrade taxonomy in the existing occurrence of swelling as features of low scale category participated in powerful relationships. Bauer et al. [53], this paper performed a programmed technique to division brain tissues from the T1WI or the "spin-lattice" dataset. The swelling mass paradigm was formed as a mesh-free MRF power reduction task, assuring communication between the atlas and the case image before the recording phase. The procedure is nonparametric, easy, and quick while preserving the same precision. In [54], locative precision weighted unseen Markov Random and maximum likelihood estimation procedure was suggested. Spatial precision, describing every pixel's locative sample precision of sampled minimum accuracy images, was presented and applied to the paradigm informing and rating. The pixels with high-incorporation precision obtain extra weight, and the opposite is true. The tumor splitting up outcomes were higher precision than employing the pixels evenly.

\section{LITERATURE SURVEY}

The principal purpose of segmentation is to convert the picture into a relevant entity, thereby enabling the image to be analyzed rapidly. At present, many brain tumor images are created in hospitals; therefore, it is unreasonable to expect such images to be segmented manually during an acceptable period; consequently, automatic segmentation is now inevitable. Segmentation MR Images of different tissue kinds are considered essential.

Cail, $\mathrm{H}$ [55] and Vermaa, R [56], the intensity power within magnetic resonance imaging pictures was applied to provide the function vector. At that juncture, the SVM was used in the categorization phase. Furthermore, they are prepared to split the okay tissue and separate the tumor and non-tumor sub-area.

James Tilton [57], they defined a method of creating superior hierarchically associated image subdivision. Such segmentations where at various levels segmentations of lesser detail could be obtained from particular area-merging algorithms. Subsequently, the area combining-based hierarchical subdivision was offered together with its recursive hierarchical segmentation. This was used to apply the information from the segmentation hierarchy according to transformations in the area attributes. Furthermore, in this method, the start spot choice in the hierarchical segmentation and the recursive hierarchical segmentation continued to challenge.

Sumitra and Saxena [58] suggested that a neural network method should be used to classify the MR brain images. These comprise three stages: extraction of attributes, dimensionality classification, and reduction. The factor removal is completed, and absolute crucial highlights, such as estimates of median, mean and variance, and highest and lowest intensity, is removed by utilizing principal component analysis (PCA) from MRI images. Furthermore, the backpropagation neural network was applied in the pattern categorization procedure. An automated method of segmenting the MRI images has been elucidated.

Xiao et al. [59], a tool was clarified to estimate tumor and brain lateral ventricular (LaV) deformation attributes. The suggested approach mainly includes four stages: Pre-processing operation, Extracting Function, Segmenting, and Classifying. During the first phase, the non-consistency and repetitive details in the surrounding picture were considered. Lateral ventricular deformation is applied to mine the characteristics, and unsupervised segmentation methods were utilized to estimate the $\mathrm{LaV}$ deformation attributes of the lesion segmentation. The most broadly applied methods are $\mathrm{K}$ nearest neighbours, with pattern matching used by conventional fuzzy connected C-mean (FCM). The main disadvantage is that the cluster CSF is wrongly assigned to a non-CSF pixel, but a comprehensive mask is utilized to remove this undesired pixel during the extraction process.

Dahsh et al. [60], suggested a mixture method, which involves three stages. Discrete wavelet transform (DWT), which is used in feature abstraction and the factor of Magnetic resonance imaging images have been reduced by applying principal-component analyses, and two algorithms have been extended. 
The principal classifier depends on the (FP-ANN), whereas another one has been utilized to categorize natural or unnatural MRI individual pictures. The disadvantages of such work are that it needs new learning datasets and a degree of adjustments in image datasets. Moreover, future work may be expanded in order to process the pathological brain tissue.

Amia Hald et al. [61], introduced an unmonitored dynamic picture segmentation utilizing fuzzy with the genetic algorithm which could segment grey-scale images automatically. This technique generally describes the spatial unmonitored grey-scale image segmentation, which separated a picture into areas. The objective of this algorithm is to provide an accurate segmentation of pictures by applying intensity information together with adjacent connections. Furthermore, the fuzzy Hopfield neural network (FHNN) grouping facilitates the creation of the population of a genetic algorithm by segmenting the images automatically in good quality.

Liwg et al. [62], suggested a new training-based multi-source mixing structure to segment brain images of newborn babies, mainly aiming effectively to assimilate features from several-source picture division performed through a random forest. In this case, the several-source, initially known as T1, T2 and FA, combines pictures which are subsequently iteratively evaluated involving potential refined tissue; for example, CSF, WM, and GM. The additional evaluation was conducted on the clinical image computing and computer secondary interference major obstacle. The technique to be used is somewhat limited and required numerous training sets with corresponding physical segmentation results. Fifty training data exist for each of the five-time focuses and much action is needed to attain manual segmentation; moreover, the limitations will be explored in future work.

Yunliang Cai et al. [63], they applied detection, structure and grouping inference for regular repetitive patterns with the pictures. Repetitive frameworks of colour patterns or repetitive reflections cause these. The segmentation algorithm advocated in this essay conformed to the traditional area growing picture segmentation project, which used an effective procedure to cluster local picture pimples into groups. The outcome of the greater-scale grouping of picture shapes may be applied to deduce objects' geometry and assess the necessary arrangement of a congested area.

Mohamad Awad et al. [64], an artificial neural network and a genetic algorithm were utilized to investigate a multicomponent segmentation of images. Various techniques were applied to separate the several-component pictures. The multi-component picture division technique was performed employing a non-parametric unmonitored ANN named Kohonen's Self-organizing map (SOM), and the mixture genetic algorithm (HGA). The image's principal features were recognized using the SOM, with HGA being utilized subsequently with no previous information to cluster the picture into homogeneous areas.

Tian LAN et al. [65] suggested a brain image technique according to the kernel FCM method. The techniques of FCM, spatial FCM, kernelled FCM and FCM are all calculated from the brain image; however, the precision element is checked with the error rate. Therefore, this essay deduces a greater precision rate in the FCM technique compared to the other methods.

Agarwal et al. [66], provided a bias field correction technique joined with the fuzzy c-means segmentation to divide the brain MRI image into two groups, GM and WM, respectively. The level set segmentation is finally applied to these areas, and this essay deduces that the results revealed a greater precision than did the other algorithms.

Ping and Honglei Wang [67], suggested an amended FCM segment the areas of an MRI brain image. This system concludes that it can increase the clusters' performance for the Gaussian and the salt and pepper degraded images.

Pratik et al. [68], Exposure and computation of brain tumor from MRI is proposed in this study. In the first step, the MRI image is turned into a grey form and reduces the noise. The gradient magnitude of input images is computed by applying both linear filtering and Sobel mask. The grey level image is changed into a topographic surface after applied the watershed segmentation and is expanded by the flooding process.

\section{CONCLUSION}

This paper illustrates several popular MRI image segmentation approaches that are extensively applied in medical image analysis. One primary aim of tumor imaging segmentation is to determine cancer precisely. Detecting brain 
tumor from MRI in a quick, precise, and increasing path is a difficult task. Brain tumor segmentation methods have presented considerable prospects in detecting and investigating tumor in medical images. This survey offers a comprehensive study of different present techniques of MRI brain tumor segmentation. It has been seen from the survey that all segmentation approach has its pros and cons. The challenging characteristics present in the brain image require further research in the segmentation field.

\section{References}

[1]. Ben Rabeh, A., Benzarti, F., \& Amiri, H. (2017). Segmentation of brain MRI using active contour model. International Journal of Imaging Systems and Technology, 27(1), 3-11.

[2]. He, S. J., Weng, X., Yang, Y., \& Yan, W. (2000, December). MRI brain images segmentation. In IEEE APCCAS 2000. 2000 IEEE Asia-Pacific Conference on Circuits and Systems. Electronic Communication Systems.(Cat. No. 00EX394) (pp. 113-116). IEEE.

[3]. Ortiz, A., Górriz, J. M., Ramirez, J., \& Salas-Gonzalez, D. (2011). MR brain image segmentation by growing hierarchical SOM and probability clustering. Electronics Letters, 47(10), 585-586.

[4]. Dawngliana, M., Deb, D., Handique, M., \& Roy, S. (2015, September). Automatic brain tumor segmentation in MRI: Hybridized multilevel thresholding and level set. In 2015 International Symposium on Advanced Computing and Communication (ISACC) (pp. 219-223). IEEE.

[5]. Neethu Ouseph, C., \& Shruti, K. A reliable method for brain tumor detection using CNN technique. In IOSR Journal of Electrical and Electronics Engineering (IOSR-JEEE), National Conference on "Emerging Research Trends in Electrical, Electronics \& Instrumentation'(ERTEEI'17).

[6]. Phillips, H. S., Kharbanda, S., Chen, R., Forrest, W. F., Soriano, R. H., Wu, T. D., ...\& Williams, P. M. (2006). Molecular subclasses of high-grade glioma predict prognosis, delineate a pattern of disease progression, and resemble stages in neurogenesis. Cancer cell, 9(3), 157-173.

[7]. Corso, J. J., Sharon, E., Dube, S., El-Saden, S., Sinha, U., \& Yuille, A. (2008). Efficient multilevel brain tumor segmentation with integrated bayesian model classification. IEEE transactions on medical imaging, 27(5), 629-640.

[8]. Rajasekaran, K. A., \& Gounder, C. C. (2018). Advanced Brain Tumor Segmentation from MRI Images. HighResolution Neuroimaging: Basic Physical Principles and Clinical Applications, 83.

[9]. Saman, S., \& Narayanan, S. J. (2019). Survey on brain tumor segmentation and feature extraction of MR images. International Journal of Multimedia Information Retrieval, 8(2), 79-99..

[10]. Olabarriaga, S. D., \& Smeulders, A. W. (2001). Interaction in the segmentation of medical images: A survey. Medical image analysis, 5(2), 127-142.

[11]. Yao, J. (2006). Image processing in tumor imaging. New techniques in oncologic imaging, 79-102.

[12].Luo S, Li R, Ourselin S. A new deformable model using dynamic gradient vector flow and adaptive balloon forces. APRS Workshop on Dig Image Comp; 2003.p. 9-14..

[13].Wong, K. P. (2005). Medical image segmentation: methods and applications in functional imaging. In Handbook of biomedical image analysis (pp. 111-182). Springer, Boston, MA.

[14].Prastawa, M., Bullitt, E., Moon, N., Van Leemput, K., \& Gerig, G. (2003). Automatic brain tumor segmentation by subject specific modification of atlas priors1. Academic radiology, 10(12), 1341-1348.

[15].Olabarriaga, S. D., \& Smeulders, A. W. (2001). Interaction in the segmentation of medical images: A survey. Medical image analysis, 5(2), 127-142.

[16].Khan, A. M., \& Ravi, S. (2013). Image segmentation methods: A comparative study.

[17].Kaur, D., \& Kaur, Y. (2014). Various image segmentation techniques: a review. International Journal of Computer Science and Mobile Computing, 3(5), 809-814.

[18]. Senthilkumaran, N., \& Vaithegi, S. (2016). Image segmentation by using thresholding techniques for medical images. Computer Science \& Engineering.

[19].Zaitoun, N. M., \& Aqel, M. J. (2015). Survey on image segmentation techniques. Procedia Computer Science, 65, 797806.national Journal, 6(1), 1-13.

[20]. Wong, K. P. (2005). Medical image segmentation: methods and applications in functional imaging. In Handbook of biomedical image analysis (pp. 111-182). Springer, Boston, MA.

[21]. Yao, J. (2006). Image processing in tumor imaging. New techniques in oncologic imaging, 79-102.

[22].Farag, A. A., Ahmed, M. N., El-Baz, A., \& Hassan, H. (2005). Advanced segmentation techniques. In Handbook of biomedical image analysis (pp. 479-533). Springer, Boston, MA.

[23].Saman, S., \& Narayanan, S. J. (2019). Survey on brain tumor segmentation and feature extraction of MR images. International Journal of Multimedia Information Retrieval, 8(2), 79-99.

[24]. Otsu, N. (1979). A threshold selection method from gray-level histograms. IEEE transactions on systems, man, and cybernetics, 9(1), 62-66.

[25].Ilhan, U., \& Ilhan, A. (2017). Brain tumor segmentation based on a new threshold approach. Procedia computer science, $120,580-587$.

[26].Taheri, S., Ong, S. H., \& Chong, V. F. H. (2010). Level-set segmentation of brain tumors using a threshold-based speed function. Image and Vision Computing, 28(1), 26-37. 
[27].Park, J. W. (2005). Connectivity-based local adaptive thresholding for carotid artery segmentation using MRA images. Image and Vision Computing, 23(14), 1277-1287.

[28].Adams, R., \& Bischof, L. (1994). Seeded region growing. IEEE Transactions on pattern analysis and machine intelligence, 16(6), 641-647.

[29].Sato, M., Lakare, S., Wan, M., Kaufman, A., \& Nakajima, M. (2000, September). A gradient magnitude based region growing algorithm for accurate segmentation. In Proceedings 2000 International Conference on Image Processing (Cat. No. 00CH37101) (Vol. 3, pp. 448-451). IEEE.

[30]. Lakare, S., \& Kaufman, A. (2000). 3D segmentation techniques for medical volumes. Center for Visual Computing, Department of Computer Science, State University of New York, 2000, 59-68.

[31].Salman, Y. M. (2009). Modified technique for volumetric brain tumor measurements. Journal of Biomedical Science and Engineering, 2(1), 16.

[32].Letteboer, M., Niessen, W., Willems, P., Dam, E. B., \& Viergever, M. (2001). Interactive multi-scale watershed segmentation of tumors in MR brain images. In Proc. of the IMIVA workshop of MICCAI.

[33].Dam, E., Loog, M., \& Letteboer, M. (2004, August). Integrating automatic and interactive brain tumor segmentation. In Proceedings of the 17th International Conference on Pattern Recognition, 2004. ICPR 2004. (Vol. 3, pp. 790-793). IEEE.

[34].Cates, J. E., Whitaker, R. T., \& Jones, G. M. (2005). Case study: an evaluation of user-assisted hierarchical watershed segmentation. Medical Image Analysis, 9(6), 566-578.

[35]. Bhattacharya, M., \& Das, A. (2008). A study on seeded region based improved watershed transformation for brain tumor segmentation. The XXIX General Assembly of the Int Union of Radio Science.

[36].Ratan, R., Sharma, S., \& Sharma, S. K. (2009). Multiparameter segmentation and quantization of brain tumor from MRI images. Indian Journal of Science and Technology, 2(2), 11-15.

[37].Gordillo, N., Montseny, E., \& Sobrevilla, P. (2013). State of the art survey on MRI brain tumor segmentation. Magnetic resonance imaging, 31(8), 1426-1438.

[38].Kong, J., Wang, J., Lu, Y., Zhang, J., Li, Y., \& Zhang, B. (2006, May). A novel approach for segmentation of MRI brain images. In MELECON 2006-2006 IEEE Mediterranean Electrotechnical Conference (pp. 525-528). IEEE.

[39].Supot, S., Thanapong, C., Chuchart, P., \& Manas, S. (2007, March). Segmentation of magnetic resonance images using discrete curve evolution and fuzzy clustering. In 2007 IEEE International Conference on Integration Technology (pp. 697-700). IEEE.

[40]. Chen S, Zhang D. Robust image segmentation using FCM with spatial constraints based on new kernel-induced distance measure. IEEE Trans Syst Man Cybern B (Cybern) 2004;34(4):1907-16.

[41].Benaichouche, A. N., Oulhadj, H., \& Siarry, P. (2013). Improved spatial fuzzy c-means clustering for image segmentation using PSO initialization, Mahalanobis distance and post-segmentation correction. Digital Signal Processing, 23(5), 1390-1400.

[42].Forouzanfar, M., Forghani, N., \& Teshnehlab, M. (2010). Parameter optimization of improved fuzzy c-means clustering algorithm for brain MR image segmentation. Engineering Applications of Artificial Intelligence, 23(2), 160168.

[43].Krinidis, S., \& Chatzis, V. (2010). A robust fuzzy local information C-means clustering algorithm. IEEE transactions on image processing, 19(5), 1328-1337.

[44].Song, J. H., Cong, W., \& Li, J. (2017). A fuzzy C-means clustering algorithm for image segmentation using nonlinear weighted local information. Journal of Information Hiding and Multimedia Signal Processing, 8(9), 1-11.

[45].Menon, N., \& Ramakrishnan, R. (2015, April). Brain tumor segmentation in MRI images using unsupervised artificial bee colony algorithm and FCM clustering. In 2015 International Conference on Communications and Signal Processing (ICCSP) (pp. 0006-0009). IEEE.

[46].Ma, M., Liang, J., Guo, M., Fan, Y., \& Yin, Y. (2011). SAR image segmentation based on Artificial Bee Colony algorithm. Applied Soft Computing, 11(8), 5205-5214.

[47].Huang, C. W., Lin, K. P., Wu, M. C., Hung, K. C., Liu, G. S., \& Jen, C. H. (2015). Intuitionisti fuzzy \$\$\$-means lustering algorithm with neighborhood attration in segmenting medial image. Soft Computing, 19(2), 459-470.

[48].Adhikari, S. K., Sing, J. K., Basu, D. K., \& Nasipuri, M. (2015). Conditional spatial fuzzy C-means clustering algorithm for segmentation of MRI images. Applied soft computing, 34, 758-769.

[49].Mahata, N., Kahali, S., Adhikari, S. K., \& Sing, J. K. (2018). Local contextual information and Gaussian function induced fuzzy clustering algorithm for brain MR image segmentation and intensity inhomogeneity estimation. Applied Soft Computing, 68, 586-596.

[50].Anguelov, D., Taskarf, B., Chatalbashev, V., Koller, D., Gupta, D., Heitz, G., \& Ng, A. (2005, June). Discriminative learning of markov random fields for segmentation of 3d scan data. In 2005 IEEE Computer Society Conference on Computer Vision and Pattern Recognition (CVPR'05) (Vol. 2, pp. 169-176). IEEE.

[51]. Capelle A, Alata O, Fernandez C, Lefevre S, Ferrie J. Unsupervised segmentation for automatic detection of brain tumors in MRI. IEEE Int Conf Image Process 2000:613-6.

[52]. Gering D, Grimson W, Kikinis R. Recognizing deviations from normalcy for brain tumor segmentation. Med Image Comput Comput Assist Interv 2002;2488:388-95. 
[53].Bauer, S., Nolte, L. P., \& Reyes, M. (2011, March). Segmentation of brain tumor images based on atlas-registration combined with a Markov-Random-Field lesion growth model. In 2011 IEEE International Symposium on Biomedical Imaging: From Nano to Macro (pp. 2018-2021). IEEE.

[54].Nie, J., Xue, Z., Liu, T., Young, G. S., Setayesh, K., Guo, L., \& Wong, S. T. (2009). Automated brain tumor segmentation using spatial accuracy-weighted hidden Markov Random Field. Computerized Medical Imaging and Graphics, 33(6), 431-441.

[55].Cai, H., Verma, R., Ou, Y., Lee, S. K., Melhem, E. R., \& Davatzikos, C. (2007, April). Probabilistic segmentation of brain tumors based on multi-modality magnetic resonance images. In 2007 4th IEEE International Symposium on Biomedical Imaging: From Nano to Macro (pp. 600-603). IEEE.

[56].Verma, R., Zacharaki, E. I., Ou, Y., Cai, H., Chawla, S., Lee, S. K., \& Davatzikos, C. (2008). Multiparametric tissue characterization of brain neoplasms and their recurrence using pattern classification of MR images. Academic radiology, 15(8), 966-977.

[57].Tilton, J. C., Tarabalka, Y., Montesano, P. M., \& Gofman, E. (2012). Best merge region-growing segmentation with integrated nonadjacent region object aggregation. IEEE Transactions on Geoscience and Remote Sensing, 50(11), 4454-4467.

[58].Sumitra, N., \& Saxena, R. K. (2013). Brain tumor classification using back propagation neural network. International Journal of Image, Graphics and Signal Processing, 5(2), 45.

[59].Xiao, K., Liang, A. L., Guan, H. B., \& Hassanien, A. E. (2013). Extraction and application of deformation-based feature in medical images. Neurocomputing, 120, 177-184.

[60].El-Dahshan, E. S. A., Hosny, T., \& Salem, A. B. M. (2010). Hybrid intelligent techniques for MRI brain images classification. Digital Signal Processing, 20(2), 433-441.

[61].Halder, A., Pramanik, S., \& Kar, A. (2011). Dynamic image segmentation using fuzzy c-means based genetic algorithm. International Journal of Computer Applications, 28(6), 15-20.

[62].Wang, L., Gao, Y., Shi, F., Li, G., Gilmore, J. H., Lin, W., \& Shen, D. (2015). LINKS: Learning-based multi-source IntegratioN frameworK for Segmentation of infant brain images. NeuroImage, 108, 160-172.

[63].Cai, Y., \& Baciu, G. (2013). Detecting, grouping, and structure inference for invariant repetitive patterns in images. IEEE Transactions on Image Processing, 22(6), 2343-2355.

[64].Awad, M., Chehdi, K., \& Nasri, A. (2007). Multicomponent image segmentation using a genetic algorithm and artificial neural network. IEEE Geoscience and remote sensing letters, 4(4), 571-575.

[65].Lan, T., Xiao, Z., Hu, C., Ding, Y., \& Qin, Z. (2014, July). MRI brain image segmentation based on Kerneled FCM algorithm and using image filtering method. In 2014 International Conference on Audio, Language and Image Processing (pp. 511-515). IEEE.

[66].Mishro, P. K., Agrawal, S., Dora, L., \& Panda, R. (2017, October). A fuzzy C-means clustering approach to HMRFEM model For MRI brain tissue segmentation. In 2017 6th International Conference on Computer Applications In Electrical Engineering-Recent Advances (CERA) (pp. 371-376). IEEE.

[67].Wang, P., \& Wang, H. (2008, December). A modified FCM algorithm for MRI brain image segmentation. In 2008 International Seminar on Future BioMedical Information Engineering (pp. 26-29). IEEE.

[68].Singhai, P. P., \& Ladhake, S. A. (2013). Brain tumor detection using marker based watershed segmentation from digital MR images. International Journal of Innovative Technology and Exploring Engineering (IJTEE) ISSN, 22783075 . 